\title{
Dyspraxia in a patient with corticobasal degeneration: the role of visual and tactile inputs to action
}

\author{
Naida L Graham, Adam Zeman, Andrew W Young, Karalyn Patterson, John R Hodges
}

\begin{abstract}
Objectives-To investigate the roles of visual and tactile information in a dyspraxic patient with corticobasal degeneration (CBD) who showed dramatic facilitation in miming the use of a tool or object when he was given a tool to manipulate; and to study the nature of the praxic and neuropsychological deficits in
\end{abstract} CBD.

Methods-The subject had clinically diagnosed CBD, and exhibited alien limb behaviour and striking ideomotor dyspraxia. General neuropsychological evaluation focused on constructional and visuospatial abilities, calculation, verbal fluency, episodic and semantic memory, plus spelling and writing because impairments in this domain were presenting complaints. Four experiments assessed the roles of visual and tactile information in the facilitation of motor performance by tools. Experiment 1 evaluated the patient's performance of six limb transitive actions under six conditions: (1) after he described the relevant tool from memory, (2) after he was shown a line drawing of the tool, (3) after he was shown a real exemplar of the tool, (4) after he watched the experimenter perform the action, (5) while he was holding the tool, and (6) immediately after he had performed the action with the tool but with the tool removed from his grasp. Experiment 2 evaluated the use of the same six tools when the patient had tactile but no visual information (while he was blindfolded). Experiments 3 and 4 assessed performance of actions appropriate to the same six tools when the patient had either neutral or inappropriate tactile feedbackthat is, while he was holding a non-tool object or a different tool.

Results-Miming of tool use was not facilitated by visual input; moreover, lack of visual information in the blindfolded condition did not reduce performance. The principal positive finding was a dramatic facilitation of the patient's ability to demonstrate object use when he was holding either the appropriate tool or a neutral object. Tools inappropriate to the requested action produced involuntary performance of the stimulus relevant action.

Conclusions-Tactile stimulation was paramount in the facilitation of motor performance in tool use by this patient with CBD. This outcome suggests that tactile information should be included in models which hypothesise modality specific inputs to the action production system. Significant impairments in spelling and letter production that have not previously been reported in CBD have also been documented.

(F Neurol Neurosurg Psychiatry 1999;67:334-344)

Keywords: ideomotor dyspraxia; corticobasal degeneration; dysgraphia

Dyspraxia is defined as a deficit in the higher order control of motor function in which the resulting impaired production of skilled movements cannot be accounted for by sensory loss, weakness, tremor, dystonia, ataxia, poor comprehension, or dementia. Since it was first described by Liepmann, ${ }^{1}$ dyspraxia has been the focus of a large body of research. Much of the earlier work examined the relation between aphasia and dyspraxia, but more recently researchers have attempted to characterise in detail different types of dyspraxia. The field has, however, been dogged by confusing and inconsistently used terminology. Ideational and ideomotor dyspraxia have been differentiated in several ways. $^{2}$ For example, ideational dyspraxia has been defined by some workers as an impairment in production of transitive gestures (those using an object, such as hammering), whereas ideomotor dyspraxia is impairment in intransitive gestures (those which do not involve an object, such as waving). ${ }^{34}$ Another way of defining these types of dyspraxia involves deficits in the use of multiple objects (ideational) compared with single objects (ideomotor). ${ }^{5}$ Because of this confusion in the use of various terms, we chose to adopt a more theoretically motivated distinction, defined in terms of deficits in the conceptual and production systems involved in tool usage. ${ }^{7-11}$ On this account, ideational dyspraxia is a disorder of the conceptual system which contains knowledge of tool functions and actions, whereas ideomotor apraxia is a disorder of the production system which includes sensorimotor action programmes concerned with the generation and control of movement.

Studies of dyspraxic patients have shown that dissociations in performance can be seen under certain testing conditions. For example, Ochipa et $a l^{12}$ documented a patient who could mime actions to command, yet could not imitate mimed actions, whereas the dyspraxic patient studied by Riddoch et $a l^{13}$ could also 
mime actions to command, but was unable to make gestures (with his right hand) corresponding to visually presented objects. Other patients with dyspraxia may be poor at miming actions, but able to perform the action when they are given the relevant tool to use, ${ }^{14-19}$ although selective impairment of tool use in association with relative preservation of ability to mime actions has also been documented..$^{20-22}$ The improvement in performance when using real objects could be explained by various mechanisms. Both De Renzi et $a l^{21}$ and Roy and Square $^{8}$ proposed that tools provide additional information which could be either perceptual (visual and/or tactile) or contextual in nature. The roles of visual and tactile information in the facilitatory effect seen with object usage have not been systematically explored, and many issues remain to be clarified, including the specific role of tactile information in praxis, as well as the nature and relative importance of visual and tactile information. Similarly, although some models hypothesise modality specific (auditory/verbal and visual) perceptual inputs to the action production system,,$^{721323-26}$ the possibility of a route from tactile input to action has received little attention.

Most studies of dyspraxia have involved patients with strokes and other focal brain injuries, although dyspraxia is also a well recognised feature of neurodegenerative diseases, including Alzheimer's disease, ${ }^{10}{ }^{14}$ progressive dyspraxia without dementia, ${ }^{27-29}$ and corticobasal degeneration. ${ }^{50-33}$ The patient we studied has probable corticobasal degeneration, which typically involves the frontoparietal cortex and basal ganglia, and produces a progressive asymmetric akinetic rigid syndrome associated with myoclonus, postural abnormalities, cortical sensory loss, and cognitive decline. Alien limb behaviour and dyspraxia are common features and indeed have been included in recent diagnostic criteria, ${ }^{303435}$ but the nature of the dyspraxia has not been explored using more theoretically motivated techniques.

The studies which have assessed praxic abilities in CBD have mostly done so as part of an investigation focused primarily on clinical issues. For example, in their study of the ability of clinicians to diagnose CBD, Litvan et $a^{\beta^{6}}$ found that ideomotor dyspraxia (as identified in neurological examination) was one of the symptoms which predicted accurate diagnosis, although they do not specify the nature of the dyspraxia. Similarly, Rinne et $a l^{30}$ found that dyspraxia is a "prominent early feature" in $\mathrm{CBD}$, and that the ideomotor form is more common than ideational dyspraxia, but the use of these terms is ambiguous. A few researchers have attempted to characterise the dyspraxia in CBD more specifically. Pillon et $a l^{11}$ showed that a group of 15 patients with CBD were impaired at tool use, as well as at miming and at imitating a mime of tool use. This occurred in the context of preserved recognition of these mimes. In their study of six patients with CBD, Jacobs et $a l^{\beta 2}$ also found severe impairment in gesture production in conjunction with preserved gesture recognition. Some researchers have suggested that the limb dyspraxia in CBD can be best characterised as limb kinetic or melokinetic dyspraxia, ${ }^{33} 37{ }^{38}$ which is defined as an inability to perform fine movements, most obvious when finger movements are assessed. ${ }^{7}$ Those who have identified this specific deficit in CBD do acknowledge, however, that it can coexist with ideational and ideomotor dyspraxia. It is worth noting that within the dyspraxia literature, the status of limb kinetic dyspraxia is controversial, with some arguing that it is not truly dyspraxia, as it can be induced in monkeys by pyramidal or corticospinal tract lesions. ${ }^{73} 39$

The most detailed study to date of the nature of the dyspraxia in CBD was done by Leiguarda et $a l^{5}$ who examined 10 patients using a test battery which incorporated production and recognition of transitive and intransitive movements, as well as multiple step sequences. Transitive movements were assessed in "either verbal, visual, or tactile modalities", and scores were summed with those obtained on intransitive movements. Results indicated that seven out of 10 patients were dyspraxic; all seven showed ideomotor dyspraxia, which was defined as impairment in production of intransitive movements, or transitive movements involving a single object. Three of the patients with ideomotor dyspraxia also showed ideational dyspraxia (defined as impairment on multiple step tasks), as well as impaired gesture comprehension. The remaining four patients with ideomotor dyspraxia exhibited normal gesture comprehension. It was therefore concluded that the dyspraxia in CBD is most likely to be ideomotor, and that it often occurs in the context of preserved knowledge about actions. But because this study collapsed together scores on transitive and intransitive movements, and did not systematically manipulate modality (for example, copying gestures $v$ producing them to verbal command, miming tool use $v$ actually using a tool, etc), it did not provide a clear picture of the nature of the dyspraxia in CBD.

This report describes our experimental study of a patient with corticobasal degeneration, who exhibited ideomotor dyspraxia (which we define as an impairment in the action production system) and alien limb behaviour. The purposes of the study were twofold. Firstly, we aimed to clarify the role of visual and tactile inputs to action by examining, in a series of experimental studies, the remarkable facilitation of motor performance shown by our patient when using real objects. These findings are considered in the context of recent cognitive models of dyspraxia. Secondly, the investigation enabled us to document, in detail, the nature of the dyspraxia, as well as the neuropsychological and clinical findings, in a patient with corticobasal degeneration.

\section{Case description}

The patient, a 61 year old right handed male chief custodian presented in September 1994 with a 9 month history of difficulty in climbing stairs, spelling, and writing. He explained that when going up stairs he had become unsure 
where to put his feet. The difficulty in writing reflected uncertainty about both the formation of individual letters and the spelling of words. These symptoms were progressive. By February 1995 two new features had emerged: his mental arithmetic had deteriorated and he had noticed a tendency for his right arm and leg to "take on a life of their own". For example, while attempting to wash his head he would find his right hand rubbing his chest; if he concentrated on a task such as writing, his right leg often rose involuntarily. At this stage he remained able to work. He had not noticed any abnormality of sensation, language, or memory. There was no relevant medical history except that he was a lifelong smoker of 20 cigarettes a day, and he had been a heavy drinker as a young man.

Neurological examination disclosed a brisk jaw jerk and a pout reflex. The patient had difficulty in working out how to climb onto the examination couch, but eventually succeeded in doing so. Similarly, he found it very difficult to comply with a request to hold his arms out straight in front of him, exhibiting involuntary movements of the right arm which had a groping exploratory character. When his arms were extended passively in front of him he was able to maintain their position for some time. Tone was increased in all four limbs with bilateral cogwheel rigidity. The right limb tendon reflexes were brisker than the left, with a right grasp reflex, and a tendency for the right hand to grope after the examiner's hand. Sensory testing showed intact appreciation of light touch and pin prick, but variable tactile inattention on the right, with normal stereognosis but poor graphaesthesia bilaterally. Severe dyspraxia for miming familiar movements (for example, using a comb), and on attempted imitation of unfamiliar movements, was rapidly apparent on simple bedside testing. Bedside cognitive testing showed normal orientation, attention (reciting the months of the year in reverse order), recall of a name and address, and spoken language abilities. Impairment of oral and written spelling and dyscalculia were evident.

The combination of progressive signs of pyramidal and extrapyramidal disorder, alien limb phenomena, and cognitive impairment including dyspraxia, dysgraphia, and dyscalculia, suggested a clinical diagnosis of corticobasal degeneration.

Routine screening for causes of progressive cognitive impairment including full blood count, erythrocyte sedimentation rate, thyroid function, syphilis serology, $B_{12}$ and folate assay, and autoantibody screen was negative. Brain CT and 3-D MRI disclosed perisylvian atrophy, involving the inferior frontal and parietal lobes, more marked on the left than the right, with high signal lesions involving the deep white matter adjacent to the posterior horns of the lateral ventricles (right $>$ left), and the corona radiata bilaterally. HMPAO-SPECT showed moderate left parietal hypometabolism.

Three years after the onset of his first symptoms the patient remained able to live alone with support from social services. He had had to give up his job, and began to find the activities of daily living increasingly effortful. At that time, he was still able to dress himself, wash, shave, and cook simple meals, although his cognitive abilities had deteriorated. The dissociation between the patient's ability to perform many of the activities of daily living and his poor performance on standard tests of praxis is mirrored in the pattern of results described below.

In the fourth year of his illness, the patient's condition declined precipitously and he became completely dependent. He remained able to feed himself and walk with a walker until about 3 years and 9 months after the onset of the first symptoms. Examination at that time indicated that his right hand was rigid and fixed in flexion. There were no spontaneous movements of the upper right limb, whereas the left arm and both legs (right leg more than the left) were almost continuously in motion. The form and execution of these movements was apparently normal, and they consisted mainly of lifting his legs in the air, or crossing and then uncrossing his legs, stroking his face with his left hand, and running his left hand up and down his trunk and legs. Assessment of muscle tone was impossible because of involuntary resistance to movement of his limbs. Speech was very perseverative, and facial expression appeared normal. Full neurological examination was not possible because of behavioural problems: the patient exhibited long outbursts of very loud and repetitive shouting. He usually repeated a short phrase such as "I'm not very well" or "Can you help me up?" The second phrase presumably refers to the fact that he was unable to remain sitting upright in bed, as the continuous motion of three of his limbs caused him to slide down into a prone position.

\section{General neuropsychological evaluation}

A battery of neuropsychological tests was administered 7 months after presentation, and twice more after 5 month and then 10 month gaps, which coincided with the assessments of praxis. The results are shown in table 1 . For tests which do not have published norms, results were compared with those of 24 age and education matched control subjects. ${ }^{40}$

At all three test sessions, the patient was orientated in time and place, and his spontaneous language was normal. Initially, the major deficits were in praxis, constructional abilities, verbal fluency, calculation, and spelling and writing. Areas of preserved performance included episodic and semantic memory, reading, and borderline visuoperceptual and spatial abilities. By the time of the third testing, the patient had developed a generalised dementia, although there were some "islands" of preserved performance: he still performed normally on naming, and on a simple visuospatial test which involved matching identical objects pictured from unusual views (table 1).

Because a problem with spelling and writing was one of the patient's presenting complaints, we investigated this in more detail. Although there is a wide degree of variation with respect 


\begin{tabular}{|c|c|c|c|c|}
\hline & \multicolumn{3}{|l|}{ Patient } & \multirow{2}{*}{$\frac{\text { Controls }}{\text { mean }(S D)}$} \\
\hline & April 95 & Sept 95 & fuly 96 & \\
\hline \multicolumn{5}{|l|}{ Constructional abilities: } \\
\hline Copy of Rey figure (36) & 10 & 14 & - & $34.0(3.0)$ \\
\hline \multicolumn{5}{|l|}{ Visuospatial abilities: } \\
\hline JLO† (30) & $21^{\star}$ & 13 & - & $27.4(4.0)$ \\
\hline VOSPt & passed all ${ }^{\star}$ subtests & $\begin{array}{l}\text { passed all* but } \\
\text { dot counting }\end{array}$ & - & - \\
\hline Object matching/unusual views (40) & $36^{\star}$ & $35^{\star}$ & $36^{\star}$ & $37.3(3.1)$ \\
\hline \multicolumn{5}{|l|}{ Calculation: } \\
\hline WAIS-R† arithmetic subtest (scaled scores) & 4 & - & - & $10 \quad(-)$ \\
\hline \multicolumn{5}{|l|}{ Episodic memory: } \\
\hline \multicolumn{5}{|l|}{ Recognition memory test $\dagger$} \\
\hline Faces $(50)$ & $42^{\star}$ & $42^{\star}$ & - & $47.3(2.8)$ \\
\hline Words (50) & $36^{\star}$ & $37^{\star}$ & 26 & $43.7(3.8)$ \\
\hline \multicolumn{5}{|l|}{ Semantic memoryt: } \\
\hline Verbal fluency 8 categories ( $1 \mathrm{~min} /$ category) & 63 & 52 & 19 & $113.7(10.5)$ \\
\hline Naming (48) & $44^{\star}$ & $44^{\star}$ & $45^{\star}$ & $43.6(2.3)$ \\
\hline Word-picture matching (48) & $48^{\star}$ & $48^{\star}$ & 40 & $47.4(1.4)$ \\
\hline Reading $(252)$ & $243^{\star}$ & - & 225 & $248.8(2.9)$ \\
\hline \multicolumn{5}{|l|}{ Spellingt: } \\
\hline Oral spelling (36) & 11 & 4 & 2 & - \\
\hline Written spelling (36) & 10 & 4 & - & $35.3(.9)$ \\
\hline \multicolumn{5}{|c|}{ Copy/transcription of single letters (each task out of 26 ): } \\
\hline lower $\rightarrow$ lower & 19 & 21 & - & $25.3(1.3)$ \\
\hline UPPER $\rightarrow$ UPPER & $25^{\star}$ & $26^{\star}$ & - & $25.9(0.3)$ \\
\hline UPPER $\rightarrow$ lower & 12 & 12 & - & $24.8(1.0)$ \\
\hline lower $\rightarrow$ UPPER & 20 & $25^{\star}$ & - & $25.4(1.3)$ \\
\hline
\end{tabular}

* Score is in normal range (within $2 \mathrm{SD}$ of control mean).

JLO =judgement of line orientation ${ }^{44}$; VOSP=visual object and space perception battery ${ }^{45}$; WAIS-R=Wechsler adult intelligence scale-revised. ${ }^{41}$ Recognition memory test ${ }^{42}$; Semantic memory tests from semantic battery ${ }^{40}$; Reading $=252$ regular and exception words ${ }^{43}$; Spelling $=36$ monosyllabic words ${ }^{46} ;-=$ not tested;

to spelling ability within the normal adult population, we think that our patient's spelling performance at presentation reflected an acquired impairment. Although we have only limited information on his premorbid spelling (which he rated as "average"), we do know that he received a good education (he attended a public (private) school until the age of 17), and he wrote regularly in his job, which included administrative duties. Spelling was assessed using a list of 36 monosyllabic words which the patient was required to write or spell orally at each of the three test sessions, and which were classified as "regular" (for example, brown, did, must) or "exception" (for example, broad, shoe, comb) on the basis of spelling to sound correspondence. ${ }^{46}$ Despite his severe dyspraxia, the patient was able to manage the motor aspects of writing at the first two (but not the third) testing sessions. When he was still able to write, his performance was equally impaired on written and oral spelling (table 1). This suggests that his problems with spelling were not simply due to problems with writing, and instead were due to a central or linguistic dysgraphia (this distinction was drawn by Ellis ${ }^{47}$ ). At the time of the first two test sessions, the patient's spelling exhibited features of surface dysgraphia $^{48}$ : he was better at spelling regular than exception words, and he tended to make phonologically plausible errors (for example, brown $\rightarrow$ BROUN, mould $\rightarrow$ MOLDE). By the third session, most of the spelling errors were not phonologically plausible, and involved letter substitutions, omissions, and additions (for example, munch $\rightarrow \mathrm{MUMCH}$, scarce $\rightarrow$ SARCE, did $\rightarrow$ DUID).

As well as his spelling problems, the patient also had an impairment at the level of writing single letters. Qualitatively speaking, his writing was somewhat slow, and he preferred to print in upper case. When each letter in the alphabet was presented to him in random order, he was able to copy virtually all upper case letters correctly, but was mildly impaired on lower case letters (table 1). The patient was also asked to transcribe letters into the alternate case (for example, $b \rightarrow B, F \rightarrow f$ ); he was significantly better at doing this when transcribing into upper case compared with lower case (session 1: $\chi^{2}=5.20, \quad \mathrm{p}<0.05 ; \quad$ session 2 : $\chi^{2}=15.83$, $\mathrm{p}<0.001)$. Most errors consisted of copying rather than transcribing stimulus letters, although a few incomplete letters were produced. The relatively worse performance on transcription, as compared with copying, of single letters seemed to be due to difficulty in remembering what specific letters look like. The patient was able to recognise letters when they were shown to him-he named all letters in both cases perfectly - but he had particular difficulty in producing letters without a model to copy.

To summarise, at the first assessment, the patient's spelling was impaired, with a preponderance of phonologically plausible spelling errors; a year further in his progression, his spelling was not only much worse overall, but most of his spelling errors were no longer phonologically plausible. The pattern he showed seemed to be an evolution from good to inadequate "control" of spelling by phonological constraints. The patient also had difficulty with writing lower (as compared to upper) case letters, especially if he had no model to copy. An association between these specific forms of central and peripheral writing impairments has been seen in other patients with neurodegenerative disease. ${ }^{46} 49$

\section{Investigation of dyspraxia}

We devised a battery of tests, based on those used by other researchers, ${ }^{71}$ to test conceptual 
Table 2 Dyspraxia test battery: assessment of the action production system. Results are reported as \% correct on each task. Details of the tasks are given in the text, and the items used are listed in the appendix. Maximum scores are shown in parentheses

\begin{tabular}{|c|c|c|}
\hline & April $1995 \mathrm{~L}, \mathrm{R}$ & May $1996 L, R$ \\
\hline \multicolumn{3}{|l|}{ Hand and finger position imitation: } \\
\hline One hand (10) & 60,0 & 0,0 \\
\hline Both hands (10) & - & 0 \\
\hline Sequences (10) & 0 & 0 \\
\hline \multicolumn{3}{|l|}{ Execution of single familiar actions: } \\
\hline \multicolumn{3}{|l|}{ Limb intransitive: } \\
\hline Command (12) & 17,0 & 8,0 \\
\hline Imitation (12) & 33,0 & 0,0 \\
\hline \multicolumn{3}{|l|}{ Limb transitive: } \\
\hline Command (12) & 25,0 & 17,0 \\
\hline Imitation (12) & 50,0 & 17,0 \\
\hline Tool (12) & 100,92 & 100,92 \\
\hline \multicolumn{3}{|l|}{ Buccofacial intransitive: } \\
\hline Command (6) & 67 & 0 \\
\hline Imitation (6) & 0 & 0 \\
\hline \multicolumn{3}{|l|}{ Buccofacial transitive: } \\
\hline Command (6) & 83 & 0 \\
\hline Imitation (6) & 100 & 0 \\
\hline Tool (6) & 100 & 67 \\
\hline \multicolumn{3}{|l|}{ Axial: } \\
\hline Command (6) & 33 & 0 \\
\hline Imitation (6) & 17 & 0 \\
\hline Execution of familiar action sequences (4) & 100 & 100 \\
\hline
\end{tabular}

$\mathrm{L}=$ left hand; $\mathrm{R}=$ right hand. order. The order of presentation of correct and incorrect mimes was counterbalanced across trials.

\section{ASSESSMENT OF THE ACTION PRODUCTION} SYSTEM (TABLE 2)

(1) Hand and finger position imitation

In this task, which consists of three subtests, the patient was asked to copy the experimenter's hand or finger positions. In the first subtest, he was asked to imitate five different static finger positions with each hand. The next subtest incorporated five static hand positions involving both hands. Finally, the patient was asked to imitate five hand movement sequences, which again involved both hands.

\section{(2) Execution of single familiar actions}

The patient was asked to perform 21 movements under the four conditions listed below. The 21 movements comprised six limb transitive, six limb intransitive, three buccofacial transitive, three buccofacial intransitive, and three axial movements. All limb movements were tested with both hands. Test items for each body part and for each action were done together, but the examiner continuously switched between conditions, and between hands (for the limb praxis items). The conditions were as follows:

(a) Verbal command-The patient was instructed, for example, "Pretend you have a toothbrush in your hand. Show me how you would use it.";

(b) Imitation-The patient was asked to copy the experimenter's mime of each action;

(c) While looking at the tool-For transitive actions only, the patient was shown a tool and instructed "Pretend you have this in your hand. Show me how you would use it.";

(d) Tool use-For transitive actions only, a tool was placed on the table in front of the patient, and he was instructed "Take this in your hand and show me how you would use it".

\section{(3) Execution of familiar action sequences}

The necessary tools and materials were placed on the table in front of the subject, and he was asked to: (1) fold a piece of paper, place it in an envelope, and seal the envelope, and (2) prepare a cup of instant coffee with milk and sugar.

The testing of action production, and subsequent experiments, was videotaped for later scoring. In view of our patient's gross impairment, we adopted a deliberately generous scoring system, using a 3 point scale $(0-2)$, with $2=$ correct; $1=$ partially correct; $0=$ incorrect. Responses coded as partially correct included some components of the target movement, but were either poorly executed (that is, clumsy or awkward movement), or incorporated one or more of the following errors: failure to account for the size of an imagined tool when miming an action (for example, placing the hand too close to the mouth when pretending to brush teeth); body part as object (for example, using a fist to represent a hammer); or inexact placement of the correct mime in space (for example, shaving his head as well as his face). 
Incorrect responses bore no similarity to an appropriate gesture, and consisted either of amorphous movements of the correct body part, or irrelevant movements of the alien limb (for example, when asked to perform actions with his right hand, the hand sometimes involuntarily went into the patient's pocket, or rubbed the top of his head). The following error types were never seen: perseveration, failure to attempt a response, and substitution of a response appropriate to another tool or action. Videotapes were scored independently by two of us on two separate occasions (NLG and $\mathrm{AZ}$ ); intrarater reliability and interrater reliability were assessed for each test (Cronbach's $\alpha$ values were between 0.78 and 1.00). In almost every instance it was unambiguous to both raters which category a particular response should be assigned to; no scoring discrepancy was greater than one point, and the few cases of these were resolved by consensus.

RESULTS

The investigation of the patient's dyspraxia showed that his knowledge of actions and tools was intact. Naming of actions was assessed twice (in April 1995 and in May 1996), and the patient achieved virtually perfect (and identical) scores on both occasions: transitive gestures 5/6; intransitive gestures $6 / 6$; axial movements $3 / 3$. Additional testing on the first occasion (only) also showed that he was able to discriminate correct from incorrect gestures (he scored 12/12 on the action recognition task), to name tools $(6 / 6)$, and to specify their use $(6 / 6)$.

By contrast, he was severely impaired at imitation of hand and finger positions, and at miming limb and axial movements to command or on imitation (table 2). The subject was unable to comply with the test condition in which he was asked to mime the use of a tool placed in front of him, as he was unable to resist manipulating objects within his reach, once they became the focus of his attention during testing. (This condition has therefore been omitted from table 2.) On tests involving finger and limb movements, scores were consistently higher for the left than the right hand, despite the patient being right handed. In fact, apart from the condition involving the use of real tools, his right hand scores were all zero; by 1996 , his left hand scores were also not a great deal better than this floor level. In addition to gross deficits in arm and hand praxis, the patient also showed some clumsiness and incoordination of fine finger movements of the type described as limb kinetic (or melokinetic) dyspraxia. ${ }^{73} 3739$

On transitive gestures, the patient's performance was markedly facilitated when he was allowed to manipulate tools. This was reflected in his normal performance on tests of everyday action sequences such as making a cup of instant coffee. Performance on tests of buccofacial praxis was initially better than on tests of limb praxis, but deteriorated substantially over the period of testing. Buccofacial tests ultimately showed the same facilitation by objects as that found with limb praxis.
We devised four further experiments to try to establish an explanation for the dissociation between the patient's poor performance on tests involving verbal command and imitation, in comparison to his near normal performance when given the opportunity to manipulate the appropriate tools. These experiments were all performed in August 1995 during two test sessions administered 1 week apart. Performance on all experiments was videotaped for later scoring. Videotapes were scored in a manner identical to that used in the assessment of the action production system, described above. Interrater reliability was assessed for each test (Cronbach's $\alpha$ values were between 0.82 and 0.92), and any disagreement in scoring was resolved by consensus. In each of the four experiments, no instruction was given to the patient regarding which hand he should use to respond. After responding with one hand, he often spontaneously attempted a response with the other hand. If he failed to do this spontaneously, and he had not provided a correct response on the first attempt, he was then asked to try with his other hand. Results from the hand which achieved the highest score on a particular test item (usually his left hand) were included in the final scores.

\section{Experiment 1}

This experiment was designed to establish which of several potential factors boosted our patient's motor performance. He was asked to mime or perform six limb transitive actions under each of six conditions. The target actions involved demonstration of the use of the following tools: hammer, pen, screwdriver, comb, razor, and toothbrush. The actions were tested in random order, whereas the order of presentation of the test conditions was fixed in the order below. The patient was asked to mime or perform the actions under the following conditions: (a) after he had generated descriptions (from memory) of the appropriate tool in as much detail as possible (condition: describe) (note that the patient provided satisfactory descriptions containing both visual and associative information); (b) after he had looked at a line drawing of the tool (condition: picture); (c) after he had seen the tool displayed beneath a glass dome (condition: vision); (d) after he had seen the examiner perform the action (condition: imitate); (e) while holding the tool (condition: tool); $(f)$ immediately after he had performed the action with the tool, but with the tool removed from his grasp (condition: continue).

This experiment confirmed that the patient's performance was facilitated by the opportunity to manipulate tools. The percentage scores in each of the test conditions was as follows: describe $25.0 \%$; picture $8.3 \%$; vision $25.0 \%$; imitate $30.0 \%$; tool $91.7 \%$; continue $0.0 \%$. It was striking that the patient was unable to continue to mime the use of a tool which he had been using quite competently before it was withdrawn from his grasp. After each tool was withdrawn, he produced amorphous movements of the relevant hand, which bore no apparent resemblance to the appropriate ges- 
ture. Looking at a picture of a tool proved counterproductive as the right hand was drawn to the pictures in an apparent attempt to manipulate the pictured objects. In all conditions of this experiment, and the subsequent experiment, performance with self directed actions was better than with externally directed actions. The percentage scores on each test item (summed across conditions) were as follows: self directed actions-combing hair $41.7 \%$, shaving $60.0 \%$, brushing teeth $41.7 \%$; externally directed actions-hammering $10.0 \%$, writing $16.7 \%$, using a screwdriver $16.6 \%$.

\section{Experiment 2}

As the presence of the appropriate tool in the patient's hand improved his performance so markedly in experiment 1 , we examined his ability to demonstrate the use of the same tools while blindfolded. Thus, in this experiment, the patient could not see the tools he manipulated. Tools were placed in his hand one at a time, and he was asked to show how he would use each one. Tools were presented in random order, and the patient proved able to recognise each one by touch. Performance was compared with a baseline condition, tested separately, but at the same test session, in which the subject was asked to demonstrate use of the same tools, but without a blindfold. Results showed that lack of visual information did not reduce his performance, as total scores were similar in the two conditions: without blindfold 8/12, with blindfold 9/12.

\section{Experiment 3}

To establish whether the precise tactile characteristics of the tool were important in facilitating the patient's performance, we asked him to mime the use of six tools (a hammer, a pen, a screwdriver, a comb, a razor, and a toothbrush) while holding five neutral objects which were not tools (a stick, a skittle, a golf ball, a brick, and a toy truck). The patient was blindfolded during this experiment, and the neutral objects were placed in his hand one at a time. He was told, for example, "Pretend that is a comb in your hand. Show me how you would use it". With the same neutral object in his hand, the patient was then asked to mime further actions, until each action had been presented. The next neutral object was then placed in his hand, and the procedure was repeated. The actions were presented in a different random order for each neutral object. Performance was compared with a baseline condition, tested separately, but at the same test session, in which the patient was asked to mime the same actions, but with nothing in his hand.

The patient was better able to perform actions while holding objects which are not tools than he was while empty handed. The mean total score for miming six actions with each of the neutral objects was $5.6 / 12$; by contrast, the score in miming the same actions while empty handed was 2.0/12. A one tailed sign test of median differences showed that the median total score for performance with each of the neutral objects was greater than the median total for performance with an empty hand $(p=0.03)$. Thus, the presence of a neutral object in the patient's hand helped to cue skilled action. The results also indicated that the patient was able to evoke some actions (using a razor and a comb) fairly consistently, yet others (hammering and writing) were never produced.

\section{Experiment 4}

Because the presence of any easily manipulable object in his hand boosted our patient's motor performance (as shown in experiment 3), we wondered how he would perform if asked to demonstrate the use of a given tool in a manner appropriate to another-for example, to hammer with a comb.

This manipulation impaired the subject's performance considerably as he sometimes could not prevent himself from performing the action appropriate to the tool he was holding, rather than the action requested. This result occurred on three occasions: when asked to use a comb as if it were a pen he combed his hair; when asked to use a pen as if it were a comb, he wrote with it; and when asked to use a razor as if it were a toothbrush he shaved his face. On two occasions (when asked to use a screwdriver like a pen and a hammer like a razor) he tried repeatedly to respond, but produced only amorphic movements. He only managed to follow the instruction on one trial (he shaved his face with a toothbrush, although the execution of this movement was clumsy). This experiment was ultimately abandoned because performance was at floor.

\section{Further clinical observations}

During these experiments we made some informal observations which may shed further light on the patient's performance.

(1) The most remarkable feature of performance was the selective facilitation of action by the manipulation of tools, which was a consistent feature throughout the period of testing. We did, however, find facilitation by other aspects of the context of action. On one occasion the patient was unable to demonstrate the use of a hammer he was holding. Supplying a nail embedded in a piece of wood enabled him to demonstrate the action successfully. Similarly, the patient sometimes had difficulty in demonstrating the use of a screwdriver until we pointed out a screw on the edge of the table at which he was sitting. He had difficulty in using scissors until we supplied him with paper. He succeeded in showing us how to "beckon" when we suggested that he pretend he was beckoning one of the experimenters standing in a corner of the room. These examples suggest that whereas "tactile" cues were potent facilitators of the patient's praxis, other aspects of the context of action, such as an appropriate target for the action, could also assist him. It is possible that his superior performance with self directed relative to externally directed actions has a similar explanation: the target of self directed actions, an important part of their context, is always present. 
(2) The patient was right handed and performed actions, such as shaving, skilfully with his right hand when the appropriate tool was made available. He also performed "automatic" actions, such as scratching himself, normally with his right hand. However, when questioned about his abilities, he consistently maintained that his left hand was now more useful than his right, and he usually used his left hand if asked to pick up or manipulate an object.

(3) "Utilisation behaviour" by the right hand was noted several times. On one occasion, for example, the patient came close to drinking a glass of water because it contained a flower he had been asked to sniff. His right hand often displayed inappropriate, involuntary exploratory behaviour, for instance when he was shown a picture of an object and asked to demonstrate its use, as in experiment 1 . His poor performance in experiment 4 could also reasonably be attributed to utilisation behaviour.

\section{Discussion}

One of the aims of this study was to document the nature of the dyspraxia in a patient with CBD. The results presented here are consistent with previous studies which have shown that ideomotor dyspraxia, defined as impairment in the action production system, and the alien limb phenomenon are common early features in CBD (see introduction). The patient had clinically diagnosed CBD, and exhibited (ideomotor) limb, axial, and buccofacial dyspraxia. The limb dyspraxia was asymmetric, with the right side being most affected. At the first assessment he was unable to mime limb or axial movements to command or imitation; performance on tests of buccofacial praxis was relatively better, although this too deteriorated over the subsequent year. Performance on tests of both limb and buccofacial praxis improved dramatically when the patient was given a tool to manipulate, as compared with miming to command (or copying the examiner's mime) of the same actions.

The other aim of this study was to investigate the roles played by tactile and visual information in facilitating performance. In experiment 1 , we examined the role of visual input. Miming of tool use was not facilitated by allowing the patient to see the examiner perform the action, or by asking him to describe the tool, look at a drawing of the tool, or even look at the tool itself. Only holding the tool had a significant beneficial impact on his ability to demonstrate its use. Moreover, once the patient had successfully performed a gesture while using a tool, he was unable to continue the movement after the tool had been removed from his grasp. This indicates that visual information was not sufficient to enable the patient to perform gestures, and points to the importance of tactile input. Experiment 2 demonstrated that removal of visual information (using a blindfold) did not affect the patient's tool use. Taken together, these results indicate that visual information had little impact on the patient's motor performance, and that tactile information was paramount.

Two further experiments manipulated the type of tactile information provided. Experiment 3 demonstrated that, for at least some actions, the tactile information provided by holding an object which was not a tool helped the patient to perform the target action successfully (for example, use a stick as a toothbrush, use a golf ball as a razor, etc). This suggests that, although his miming of tool use was facilitated by tactile information, the information required can be rather non-specific, as some of the objects did not approximate to the shape of the real tool associated with the requested action. In the final experiment, we found that the patient was unable to mime the use of a given tool in a manner appropriate to another tool (for example, using a comb as if it were a pen). The patient identified each tool placed in his hand, and was then unable to prevent himself from using each one in the manner appropriate to the tool, rather than in the manner instructed.

The finding that dyspraxic motor performance can be boosted by tactile information could be accommodated readily by models which hypothesise modality specific inputs to the action production system. ${ }^{7}{ }^{12} 3^{23-26}$ As stated in the introduction, these models typically include visual and auditory $\backslash$ verbal inputs, which are included to explain facilitation or detriment to dyspraxic motor performance under certain conditions. Such frameworks typically do not include tactile input, although there are exceptions. ${ }^{76}$ The results presented here, which provide the first demonstration of a specific tactile enhancement of dyspraxic motor performance, indicate that there is a tactile route to action production which can be selectively preserved.

This conclusion is further supported by the finding that the tactile route to action can be selectively damaged. The performance of two of the dyspraxic patients studied by De Renzi et $a l^{21}$ was more impaired when they handled objects than when they mimed their use. This pattern has been documented by other researchers (Motomura and Yamadori, ${ }^{20}$ Klein, 1924, cited in De Renzi et $a l^{11}$ and case 8 of Brown $\left.^{50}\right)$. Motomura and Yamadori ${ }^{20}$ speculated that tool use may be regulated by "a special class of neurons"; they noted a parallel with research carried out with monkeys ${ }^{51}$ that documented evidence for "hand-movement-related neurons", located in the posterior parietal cortex, which fire only when monkeys use real tools.

There are at least two mechanisms by which tactile information could facilitate gesturing in dyspraxic patients. Firstly, holding onto an object obviously provides kinaesthetic feedback, which may support either the correct posture or hand position for a gesture. It has been shown that (involuntary) postural adjustments are made before arm movements, ${ }^{52}$ and it has been suggested that kinaesthetic information may help to establish "the postural context for the gesture". ${ }^{26}$ Similarly, it may be that holding onto an object enabled our patient 
to get his hand into the correct orientation, and provided mechanical support to keep his hand in the correct posture, thereby enabling him to perform the gesture successfully. Because he was unable to continue with actions immediately after the relevant tool had been removed from his grasp, it seems that being in the correct posture for a gesture was not sufficient to enable him to execute it. We conclude that the mechanical support provided by holding the appropriate tool (or a neutral object) is crucial. It seems, however, that the exact hand position (or aperture of grip) is not critical, as performance often improved when holding objects which differed in size and shape from the object which matched the gesture. This effect is not entirely non-specific, as he was completely unable to perform the correct mime when holding another gesture specific tool (see experiment 4).

The second mechanism by which using a tool could facilitate gesture performance pertains to context. Handling a tool provides a more natural context for a gesture than does miming the use of a tool. The importance of context in the motor performance of dyspraxic patients has received much attention, and may explain why deficits are often more apparent in context-impoverished formal testing than in everyday life. ${ }^{78185354}$ The contribution of context to our patient's motor performance is supported by several lines of evidence: he showed preservation of the activities of daily living at a time when testing clearly demonstrated dyspraxia; his performance was facilitated by having a tool to use, as well as by other aspects of the context of action, such as being given the recipient object of an action (for example, he had difficulty in using scissors until he was given paper to cut); he had an alien limb, which could be interpreted as a manifestation of pathological context dependence. Alien limb behaviour is characterised by involuntary context driven actions, such as groping and utilisation behaviour. ${ }^{55}$ It could also be argued that the patient's relatively well preserved ability to perform self directed actions results from the fact that the target of such actions, an important part of their context, is always present. A complete adjudication between these interpretations clearly requires further research.

The present study also provides support for a competence/performance distinction in dyspraxia. Roy and Square ${ }^{811}$ proposed two subsystems involved in praxis: the conceptual system includes knowledge of tools and actions, whereas the production system is concerned with the execution of action. Our patient was able to identify gestures which he was unable to perform, a finding consistent with preservation of the conceptual system in conjunction with impairment in the action production system. This pattern of performance has been found in other patients with CBD. ${ }^{5132}$ Rapcsak et al ${ }^{9}$ documented preserved conceptual knowledge of action in a dyspraxic patient who had progressive biparietal atrophy; they noted that this pattern is seen relatively rarely because the two action subsys- tems are often damaged concurrently due to their neuroanatomical proximity. Furthermore, dyspraxic patients are often aphasic, making assessment of the conceptual subsystem more difficult.

The preservation of the conceptual system for action in our patient is consistent with the neuropsychological test results, which disclosed relatively preserved semantic memory, even after 15 months of follow up. At that time he showed relatively well preserved naming and word-picture matching, in the context of a fairly generalised dementia. Although the cognitive decline in CBD is not always emphasised, ${ }^{56}{ }^{57}$ most recent studies acknowledge that it is a common feature in this clinicopathological syndrome. ${ }^{31} 3658$ The results of our patient's neuropsychological testing are consistent with those of other patients with $\mathrm{CBD}^{31} 3359$ in that he showed prominent deficits in verbal fluency, calculation, constructional abilities, and praxis, borderline visuospatial skills, but preserved recognition memory (on the first two occasions he was tested) and naming. An additional feature not previously reported is that the patient presented with a severe spelling impairment and problems in letter production which consisted of difficulty in writing single letters without a model to copy. Because spelling has not been extensively studied in CBD, it is not yet possible to predict whether most patients with this disorder should be expected to have impaired spelling. Difficulty with writing has, however, often been found. 30333557 This is to be expected, as dyspraxia is a major symptom of CBD. Given the severity of his dyspraxia, it is remarkable that our patient was able to write as well as he did. It has been suggested that dyspraxic dysgraphia is a symptom of a more general motor programming deficit, and is therefore "an inevitable concomitant of limb apraxia". ${ }^{60}$ We have shown that it is possible for the motor aspects of writing to be preserved in the context of limb dyspraxia, although ultimately our patient's dyspraxia prevented him from writing. This dissociation has been reported previously, ${ }^{60}$ but rarely.

In conclusion, we studied the nature of the dyspraxia in a patient with probable CBD, and found (as others have) that our patient exhibited asymmetric ideomotor dyspraxia in the context of preserved conceptual knowledge about action. In addition, the patient was better at using tools than at miming or imitating mimes of their use. Investigation of this phenomenon provided support for models of praxis which hypothesise modality-specific inputs to an action production system. Specifically, we found evidence for a tactile route to action which can be selectively preserved in dyspraxia. The existence of CBD as a distinct clinicopathological syndrome has been disputed, although it seems to be gaining wider acceptance; the results presented here add to the growing body of evidence for a distinctive syndrome.

We thank the patient for his generous participation in this study. The research was supported by a Medical Research Council studentship to NLG, and a Medical Research Council project grant to JRH. 
Appendix

Lists of items used in assessments of the conceptual system for action and the action production system

(1) The items below were used in the following tasks: naming of actions (limb and axial items only), naming of tools (limb transitive items only), specification of tool use (limb transitive items only) and execution of single familiar actions (all items).

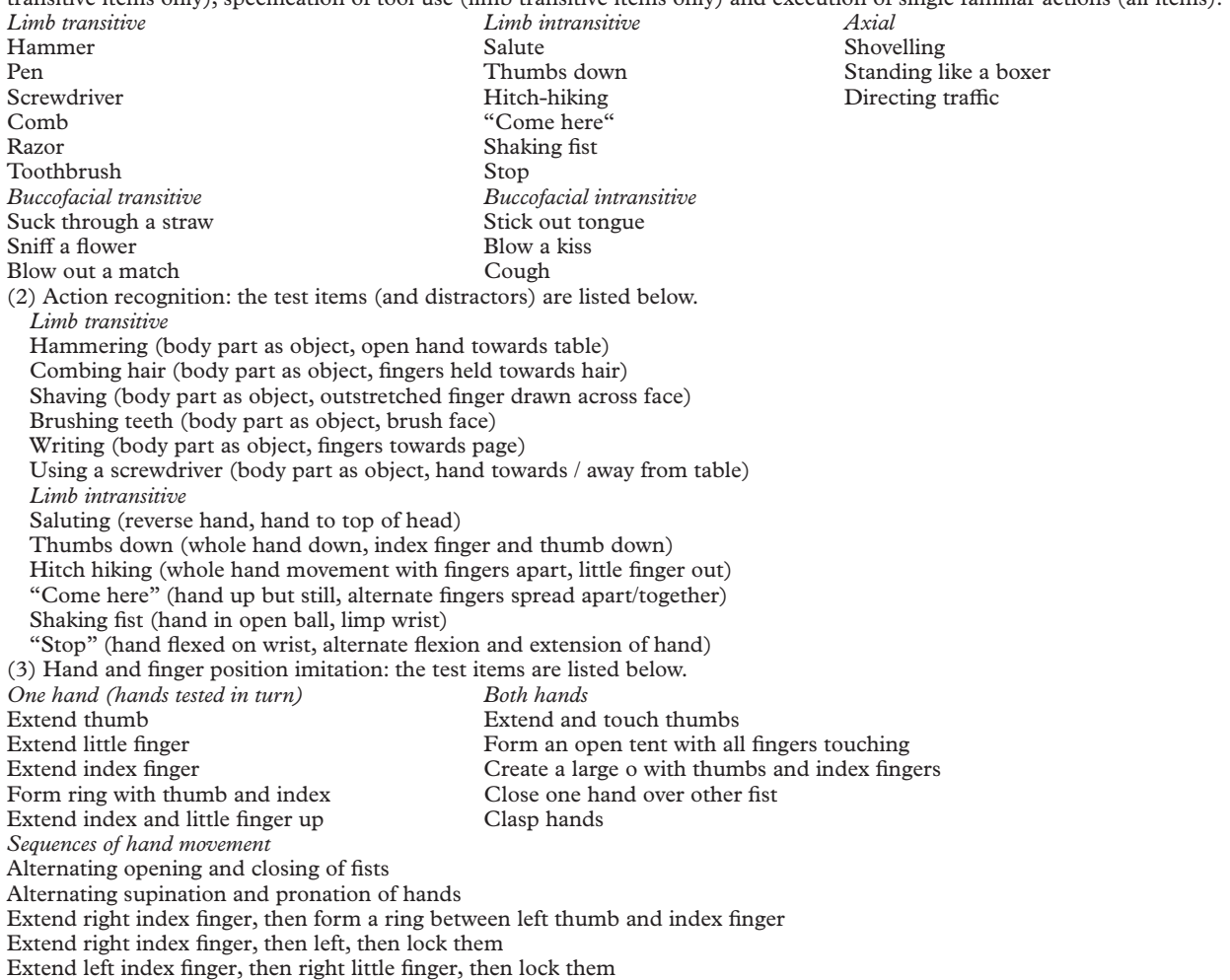

Extend left index finger, then right little finger, then lock them

1 Liepmann H. Das Krankheitsbild der Apraxie (Motorische Asymbolie) Monatschrift fur Psychiatrie und Neurologie, 8, 15-44. Translated in 1977 as: the syndrome of apraxia (motor asymboly) based on a case of unilateral apraxia. In: Rottenberg DA, Hochberg FH, ed. Neurological classics in modern translation. New York: Macmillan, 1900:155-83.

2 Mozaz MJ. Ideational and ideomotor apraxia: a qualitative analysis. Behavioural Neurology 1992;5:11-17.

3 De Renzi E, Pieczuro A, Vignolo LA. Ideational apraxia: a quantitative study. Neuropsychologia 1968;6:41-52.

4 Agostini E, Colett A, Orlando G, et al. Apraxia in deep cerebral lesions. 7 Neurol Neurosurg Psychiatry 1983;46:804-8.

5 Leiguarda R, Lees AJ, Merello M, et al. The nature of apraxia in corticobasal degeneration. $\mathcal{F}$ Neurol Neurosurg Psychiatry 1994;57:455-9.

6 Ochipa C, Rothi LJG, Heilman KM. Ideational apraxia: a deficit in tool selection and use. Ann Neurol 1989;25:190deficit

7 Heilman KM, Rothi LJG. Apraxia. In: Heilman KM, Valenstein E, eds. Clinical neuropsychology. 3rd ed. Oxford: Oxford University Press, 1993:141-63.

8 Roy EA, Square PA. Neuropsychology of movement sequencing disorders and apraxia. In: Zaidel DW, ed. Neuropsychology. London: Academic Press, 1994:183-218.

9 Rapcsak SZ, Ochipa C, Anderson KC, et al. Progressive ideomotor apraxia: evidence for a selective impairment of the action production system. Brain Cogn 1995;27:213-36.

10 Ochipa C, Rothi LJG, Heilman KM. Conceptual apraxia in Alzheimer's disease. Brain 1992;115:1061-71.

11 Roy EA, Square PA. Common considerations in the study of limb, verbal and oral apraxia. In: Roy EA, ed. Neuropsychological studies of apraxia an

12 Ochipa C, Rothi LJG, Heilman KM. Conduction apraxia. $\mathcal{F}$ Neurol Neurosurg Psychiatry 1994;57:1241-4.

13 Riddoch MJ, Humphreys GW, Price CJ. Routes to action: evidence from apraxia. Cognitive Neuropsychology 1989;6: 437-54.

14 Rapcsak SZ, Croswell SC, Rubens A. Apraxia in Alzheimer's disease. Neurology 1989;39:664-8.

15 McDonald S, Tate RL, Rigby J. Error types in ideomotor apraxia: a quantitative analysis. Brain Cogn 1994;25:250270.

16 Pilgrim E, Humphreys GW. Impairment of action to visua objects in a case of ideomotor apraxia. Cognitive Neuropsychology 1991;8:459-73.

17 Pilgrim E, Humphreys GW. Rehabilitation of a case of ideomotor apraxia. In: Riddoch MJ, Humphreys GW, eds. Cog-
nitive neuropsychology and cognitive rehabilitation. Hove, UK: nitive neuropsychology and cognitive reh
Lawrence Erlbaum 1994:271-85.
18 Schnider A, Hanlon RE, Alexander DN, et al. Ideomotor apraxia: behavioral dimensions and neuroanatomical basis. apraxia: behavioral dimensions

19 Rapcsak SZ, Ochipa C, Beeson PM, et al. Praxis and the right hemisphere. Brain Cogn 1993;23:181-202.

20 Motomura N, Yamadori A. A case of ideational apraxia with impairment of object use and preservation of object pantomime. Cortex 1994;30:167-70.

21 De Renzi E, Faglioni P, Sorgato P. Modality-specific and supramodal mechanisms of apraxia. Brain 1982;105:30112 .

22 Motomura N, Yamadori A, Asaba $\mathrm{H}$, et al. Failure to manipulate objects secondary to active touch disturbance. Cortex 1990;26:473-7.

23 Rothi LJG, Ochipa C, Heilman KM. A cognitive neuropsychological model of limb praxis. Cognitive Neuropsychology $1991 ; 8: 443-58$.

24 Rothi LJG, Ochipa C, Heilman KM. A cognitive neuropsychological model of limb praxis and apraxia. In: Rothi LJG, Heilman KM, eds. Apraxia: the neuropsychology of action. Hove, UK: Psychology Press, 1997:29-49.

25 Roy EA. Hand preference, manual asymmetries, and limb apraxia. In: Elliott D, Roy EA, eds. Manual asymmetries in motor performance. New York: CRC Press, 1996:215-36.

26 Roy EA, Hall C. Limb apraxia: a process approach. In: Proteau L, Elliott D, ed. Vision and motor control. Amsterdam: Elsevier, 1992:261-82.

27 De Renzi E. Slowly progressive visual agnosia or apraxia without dementia. Cortex 1986;22:171-80.

28 Dick JPR, Snowden J, Northen B, et al. Slowly progressive apraxia. Behavioural Neurology 1989;2:101-14.

29 Fukui T, Sugita K, Kawamura M, et al. Primary progressive apraxia in Pick's disease: a clinicopathologic study. Neurology 1996;47:467-73.

30 Rinne JO, Lee MS, Thompson PD, et al. Corticobasal degeneration. Brain 1994;117:1 183-96.

31 Pillon B, Blin J, Vidailhet $M$, et al. The neuropsychological pattern of corticobasal degeneration: comparison with progressive surpranuclear palsy and Alzheimer's disease. Neurology 1995;45:1477-83.

32 Jacobs DH, Boston MA, Adair JC, et al. Apraxia in corticobasal degeneration (CBD). Neurology 1995;45 (suppl 4):266-67.

33 Moreaud O, Naegele B, Pellat J. The nature of apraxia in corticobasal degeneration: a case of melokinetic apraxia. Neuropsychiatry, Neuropsychology, and Behavioral Neurology 1996;9:288-92.

34 Lang AE, Riley DE, Bergeron C. Cortical-basal ganglionic degeneration. In: Calne DB, ed. Neurodegenerative diseases. Philadelphia: WB Saunders, 1994:877-894. 
35 Riley DE, Lang AE, Lewis MB, et al. Cortical-basal ganglionic degeneration. Neurology 1990;40:1203-12.

36 Litvan I, Agid Y, Goetz C, et al. Accuracy of the clinical diagnosis of corticobasal degeneration: a clinicopathologic study. Neurology 1997;48:119-25.

37 Okuda B, Tachibana $\mathrm{H}$. The nature of apraxia in corticobasal degeneration [letter]. I Neurol Neurosurg Psychiatry 1994;57:1548-9.

38 Okuda B, Tachibana $\mathrm{H}$, Takeda M, et al. Focal cortical hypoperfusion in corticobasal degeneration demonstrated by three-dimensional surface display with ${ }^{123}$ I-IMP: a possible cause of apraxia. Neuroradiology 1995;37:642-4

39 Leiguarda R, Merello M, Starkstein S, et al. Reply to Okuda and Tachibana [letter]. F Neurol Neurosurg Psychiatry 1994; 57:1549.

40 Hodges JR, Patterson K. Is semantic memory consistently impaired early in the course of Alzheimer's disease? Neuroanatomical and diagnostic implications. Neuropsychologia 1995;33:441-59.

41 Wechsler DA. Wechsler adult intelligence scale - revised. New York: Psychological Corporation, 1981

42 Warrington EK Recognition memory test. Windsor: NFER Nelson, 1984

43 Patterson K, Hodges JR. Deterioration of word meaning: implications for reading. Neuropsychologia 1992;30:102540 .

44 Benton AL, deS Hamsher K, Varney NR, et al. Fudgement of line orientation. New York: Oxford University Press, 1983.

45 Warrington EK, James M. The visual object and space perception battery. Bury St Edmunds: Thames Valley Test Company, 1991.

46 Graham NL, Patterson K, Hodges JR. Progressive dysgraphia: co-occurrence of central and peripheral impairments. Cognitive Neuropsychology 1997;14:9751005.

47 Ellis AW. Normal writing processes and peripheral acquired dysgraphias. Language and Cognitive Processes 1988;3:99127.
48 Beauvois MF, Derouesné J. Lexical or orthographic agraphia. Brain 1981;104:21-49.

49 Hughes JC, Graham N, Patterson K, et al. Dysgraphia in mild dementia of Alzheimer's type. Neuropsychologia 1997; 35:533-45.

50 Brown JW. Aphasia, apraxia and agnosia. Springfield, Ill: Charles C Thomas, 1972.

51 Taira M, Mine S, Georgopoulos AP, et al. Parietal cortex neurons of the monkey related to the visual guidance of hand movement. Exp Brain Res 1990;83:29-36.

52 Frank JS, Earl M. Coordination of posture and movement. Physical therapy 1990;70:855-63.

53 Cubelli R, Della Sala S. The legacy of automatic/voluntary dissociation in apraxia. Neurocase 1996;2:449-54.

54 De Renzi E, Motti F, Nichelli P. Imitating gestures: a quantitative approach to ideomotor apraxia. Arch Neurol 1980;37:6-10.

55 Della Sala S, Marchetti C, Spinnler M. The anarchic hand: a fronto-mesial sign. In: Boller F, Grafman J, ed. Handbook of neuropsychology. Amsterdam: Elsevier, 1994;9:233-55.

56 Riley DE, Lang AE. Corticobasal ganglionic degeneration (CBGD): further observations in six additional cases [abstract]. Neurology 1988;38(suppl 1):360.

57 Rebeiz JJ, Kolodny EH, Richardson EP. Corticodentatonigral degeneration with neuronal achromasia. Arch Neurol 1968;18:20-33.

58 Beatty WW, Scott JG, Wilson DA, et al. Memory deficits in a demented patient with probable corticobasal degeneration. F Geriatr Psychiatry Neurol 1995;8:132-6.

59 Massman PJ, Kreiter KT, Jankovic J, et al. Neuropsychological functioning in cortical-basal ganglionic degeneration: differentiation from Alzheimer's disease. Neurology 1996; 46:720-6.

60 Coslett HB, Rothi LJG, Valenstein E, et al. Dissociations of writing and praxis: two cases in point. Brain Lang 1986;28: 357-69. 